\title{
Paideia: relatório de gestão - 2009
}

\author{
Manoel Antônio dos Santos \\ Universidade de São Paulo, Ribeirão Preto-SP, Brasil
}

Este relatório tem por objetivo descrever a gestão editorial da revista Paideia no ano de 2009. Este é um modo de consolidar o compromisso de tornar público o processo de tramitação dos manuscritos avaliados pela revista no decorrer do referido período. A sistematização das informações relativas ao processo editorial permite situar a relevância e abrangência da Paideia no contexto das publicações científicas na área das Ciências Humanas, sobretudo da Psicologia e Educação. Parte-se do pressuposto de que a produção científica não reflete apenas um conjunto de conhecimentos aferidos por rigorosa revisão de pares, mas implica também um corpo de pesquisadores que reproduzem e, vez ou outra, transgridem, o modus operandi da ciência.

O presente relatório está subdividido nas seguintes seções: Espaço editorial e critérios de arbitragem, Processo editorial - 2009 e Considerações finais.

\section{Espaço editorial e critérios de arbitragem}

A Paideia é publicação quadrimestral do Programa de Pós-graduação em Psicologia, da Faculdade de Filosofia, Ciências e Letras de Ribeirão Preto da Universidade de São Paulo (FFCLRP-USP). Tem como escopo e foco editorial publicar trabalhos relacionados à área da Psicologia, Educação e afins. Os textos publicados devem enquadrar-se nas categorias: relato de pesquisa, estudo teórico, relato de experiência profissional, revisão crítica da literatura, comunicação breve, nota técnica e resenha. Fundada em 1991, a revista já publicou 45 fascículos, marcados pela diversidade e abrangência dos temas discutidos por seus artigos, devido ao amplo campo de conhecimento circunscrito pela Psicologia e Educação e à sua política editorial, aberta às diversas visões e tendências contemporâneas.

No que concerne às fontes de financiamento, é preciso destacar que, de forma regular e sistemática, a Paideia tem contado com recursos financeiros advindos do Programa de Pós-graduação em Psicologia da FFCLRP-USP, do Programa de Apoio às Publicações Científicas Periódicas da USP-SIBI e do Programa de Apoio a Publicações Científicas-CNPq.

Ao final de cada fascículo constam as normas de publicação, disponíveis em três idiomas: português, inglês e

\footnotetext{
1 Endereço para correspondência:
}

Prof. Dr. Manoel Antônio dos Santos. Universidade de São Paulo. Faculdade de Filosofia, Ciências e Letras de Ribeirão Preto. Departamento de Psicologia e Educação. Av. Bandeirantes, 3900. CEP: 14040-901. Ribeirão Preto-SP, Brasil. E-mail: masantos@ffclrp.usp.br espanhol. O último fascículo de cada volume contempla a lista de assessores ad hoc que revisaram manuscritos durante $\mathrm{o}$ ano vigente.

A revista é distribuída gratuitamente para aproximadamente 160 bibliotecas de universidades públicas brasileiras vinculadas a cursos de graduação em Psicologia e/ou Educação, além de alguns países do exterior, como Portugal, Espanha, México e EUA. Isso significa que por três vezes ao ano são enviados exemplares para universidades e faculdades de diversos estados brasileiros e algumas do exterior, bem como para Programas de Pós-graduação nas áreas de Psicologia e Educação. Também são estabelecidas permutas com periódicos brasileiros e internacionais. O último exemplar de cada volume também é encaminhado a todos os consultores ad hoc que colaboraram com a revista durante o ano.

Atualmente, a Paideia está indexada nas seguintes bases de dados: (a) SciELO - Scientific Electronic Library Online (Fundação de Amparo à Pesquisa do Estado de São Paulo/ Centro Latino-Americano e do Caribe de Informação em Ciências da Saúde); (b) PsycINFO (American Psychological Association); (c) LILACS - Literatura Latino-Americana e do Caribe em Ciências da Saúde (Centro Latino-americano y del Caribe de Informacion en Ciencias de la Salud); (d) CLASE-Citas - Latinoamericanas en Ciências Sociales y Humanidades (Universidad Nacional Autónoma de México); (e) PSICODOC (Colégio Oficial de Psicólogos de Madrid/Universidad Complutense de Madrid); (f) Ulrich's Internacional Periodicals Directory; (g) Catálogo Latindex; (h) Index-Psi Periódicos (Conselho Federal de Psicologia/Pontifícia Universidade Católica de Campinas). Em 2009 a revista obteve a indexação nas bases internacionais Ulrich's International Periodicals e Catálogo Latindex.

A Comissão Editorial têm trabalhado exaustivamente para que os antigos fascículos da revista Paideia sejam disponibilizados no site da SciELO, a partir da conversão dos arquivos para o padrão utilizado por esta base indexadora de textos completos. Lembramos que, em 2008, por meio de recursos captados no SIBI-USP, foi possível digitalizar os fascículos 13(26), 2003; 14(27), 14(28), 14(29), 2004; 15(30), 15(31), 15(32), 2005; e 16(33), 2006, e incluí-los no sistema SciELO nos primeiros meses de 2009, ampliando o acesso ao conteúdo já publicado pela Paideia.

Graças ao aporte de recursos complementares do SIBI-USP, em 2009, foi possível ampliar o acervo disponível na base SciELO, agregando mais 12 fascículos publicados anteriormente: 8(14-15), 1998; 9(16), 9(17), 1999; 10(18), 10(19), 2000; 11(20), 11(21), 2001; 12(22), 12(23), 12(24), 2002; 13(25), 2003. Desse modo, ao completar seus 19 anos 
de existência, em 2009, a Paideia conseguiu disponibilizar os conteúdos integrais publicados em seus últimos 12 anos, ampliando a visibilidade da revista.

No que tange aos critérios do Qualis de Periódicos, área Psicologia, Paideia tem sido avaliada desde 1998 pela comissão conjunta CAPES (Coordenação de Aperfeiçoamento de Pessoal de Nível Superior)/ANPEPP (Associação Nacional de Pesquisa e Pós-graduação em Psicologia). Manteve nas classificações passadas o conceito A-Nacional, conforme os critérios de normalização, publicação, circulação, autoria, conteúdo e gestão editorial vigentes à época.

A partir de 2008, foram adotadas por esta comissão novas diretrizes de avaliação das publicações científicas (Bastos e cols., 2009). Além dos critérios mínimos, que compreendem padrões formais e de arbitragem, considerou-se, sobretudo, a presença e qualidade dos indexadores em que os periódicos estão registrados. De acordo com esses critérios, as revistas foram hierarquizadas em estratos (A1, A2; B1, B2, B3, B4, B5) a partir do exame dos requisitos que cada base de dados contempla para indexação. Diante disto, a revista Paideia foi classificada no estrato B1, que contempla periódicos indexados no ISI, ou PsycINFO, ou Scopus, ou SciELO; ou em quatro ou mais dos seguintes: CLASE, Latindex, LILACS, PSICODOC, PASCAL ou Redalyc.

Cabe ressaltar que as atuais diretrizes fixadas pela comissão CAPES-ANPEPP constituem etapa de transição, ou seja, visam incentivar as revistas da área a se adequarem aos requisitos estabelecidos, ao mesmo tempo em que permitem à comunidade científica discutir suas consequências, que poderão redundar em uma desejável revisão. A diversidade de subáreas que compõem o campo da Psicologia e que fazem interface com outras áreas do conhecimento, tais como: ciências biológicas, ciências humanas e artes, traduzem-se nas peculiaridades de seus periódicos, consistindo grande desafio para a constituição de critérios norteadores para a avaliação (Bastos e cols., 2009).

No que tange ao processo de arbitragem dos manuscritos, Paideia adota o mecanismo de revisão cega e dupla por pares de reconhecido saber da comunidade científica, ou seja, tanta a identidade dos autores quanto dos assessores ad hoc é mantida em sigilo. Os trabalhos submetidos à avaliação devem estar de acordo com as orientações das normas de publicação e são aceitos ou recusados pela Comissão Editorial com base nas recomendações de seus membros e dos consultores ad hoc designados para aprimorar o processo avaliativo.

\section{Processo editorial - 2009}

No que se refere ao processo editorial da Paideia no ano de 2009, o tempo médio de tramitação dos manuscritos foi de, aproximadamente, seis meses. Neste ano, a revista recebeu 101 manuscritos novos. Do total de artigos em avaliação no período em apreço, 74 foram aprovados, 50 recusados, nove tiveram sua tramitação suspensa pelos autores e 94 ainda estavam em processo de avaliação em janeiro de 2010, ocasião em que os dados foram compilados para a redação deste relatório (Tabela 1). Considerando os trabalhos cujo processo editorial foi encerrado em $2009(n=133)$, a revista aprovou $55,6 \%$ e recusou $37,6 \%$.

Tabela 1

Situação editorial dos manuscritos em avaliação pela Paideia em 2009

\begin{tabular}{lcc}
\hline \multicolumn{1}{c}{ Parecer final } & n & \% \\
\hline Aprovado & 74 & 32,6 \\
Recusado & 50 & 22,03 \\
Interrompido & 9 & 3,96 \\
Em processo editorial & 94 & 41,41 \\
Total & 227 & 100 \\
\hline
\end{tabular}

Em 2009, no volume 19, fascículos 42, 43 e 44, foram publicados 39 artigos, uma nota técnica e uma comunicação breve, além de três resenhas, três editoriais e um relatório de gestão. Os trabalhos publicados enquadraram-se nas seguintes categorias: (a) relato de pesquisa $(36,81,8 \%)$; (b) estudo teórico (um, 2,3\%); (c) revisão crítica da literatura (uma, 2,3\%); (d) relato de experiência profissional (uma, 2,3\%); (e) comunicação breve (uma, 2,3\%), (f) nota técnica (uma, 2,3\%), (g) resenhas (três, 6,8\%), como evidencia a Tabela 2 .

Tabela 2

Categoria dos manuscritos publicados na Paideia em 2009

\begin{tabular}{lcc}
\hline \multicolumn{1}{c}{ Tipo de trabalho } & n & \% \\
\hline Relato de pesquisa & 36 & 81,8 \\
Estudo teórico & 1 & 2,3 \\
Revisão da literatura & 1 & 2,3 \\
Relato de experiência & 1 & 2,3 \\
Comunicação breve & 1 & 2,3 \\
Nota técnica & 1 & 2,3 \\
Resenha & 3 & 6,8 \\
Total & 44 & 100 \\
\hline
\end{tabular}

No que concerne à filiação institucional dos autores, Paideia publicou artigos provenientes de 28 universidades brasileiras, com predomínio da Universidade de São Paulo e Pontificia Universidade Católica. São instituições de ensino superior de 12 estados brasileiros. Também vieram a lume artigos internacionais oriundos de seis universidades estrangeiras, a saber: Maltepe University, Turquia; Uludag University, Turquia; Université de Bourgogne, França; Universidad de La Frontera, Chile; Universidade do Minho, Portugal; Syracuse University, Estados Unidos da América (Tabela 3). 
Tabela 3

Origem institucional dos artigos publicados na revista Paideia em 2009*

\begin{tabular}{|c|c|c|}
\hline Instituições Nacionais & $n$ & $\%$ \\
\hline Universidade de São Paulo-SP & 9 & 18 \\
\hline Pontifícia Universidade Católica-SP, RJ, RS & 5 & 10 \\
\hline Universidade de Brasília-DF & 4 & 8 \\
\hline Universidade Federal de São Carlos-SP & 3 & 6 \\
\hline Universidade Federal de Santa Catarina-SC & 3 & 6 \\
\hline Universidade Federal do Rio de Janeiro-RJ & 2 & 4 \\
\hline Universidade Federal do Espírito Santo-ES & 2 & 4 \\
\hline $\begin{array}{l}\text { Universidade Estadual Paulista Júlio de } \\
\text { Mesquita Filho-SP }\end{array}$ & 2 & 4 \\
\hline Universidade Federal de São Paulo-SP & 1 & 2 \\
\hline Universidade Federal do ABC-SP & 1 & 2 \\
\hline Universidade Presbiteriana Mackenzie-SP & 1 & 2 \\
\hline Universidade Estadual de Campinas-SP & 1 & 2 \\
\hline Universidade do Sagrado Coração-SP & 1 & 2 \\
\hline Universidade Federal de Juiz de Fora-MG & 1 & 2 \\
\hline Universidade Federal do Triângulo Mineiro-MG & 1 & 2 \\
\hline Universidade Estácio de Sá-RJ & 1 & 2 \\
\hline Universidade do Estado do Rio de Janeiro-RJ & 1 & 2 \\
\hline $\begin{array}{l}\text { Universidade Comunitária Regional de } \\
\text { Chapecó-SC }\end{array}$ & 1 & 2 \\
\hline Universidade Federal do Paraná-PR & 1 & 2 \\
\hline Universidade Federal do Rio Grande do Sul-RS & 1 & 2 \\
\hline Universidade Federal do Rio Grande-RS & 1 & 2 \\
\hline $\begin{array}{l}\text { Universidade Regional Integrada do Alto } \\
\text { Uruguai e das Missões-RS }\end{array}$ & 1 & 2 \\
\hline Universidade do Vale do Rio dos Sinos-RS & 1 & 2 \\
\hline $\begin{array}{l}\text { Universidade Federal do Rio Grande } \\
\text { do Norte-RN }\end{array}$ & 1 & 2 \\
\hline Universidade Federal da Bahia, BA & 1 & 2 \\
\hline Universidade Católica de Salvador-BA & 1 & 2 \\
\hline Universidade Católica de Pernambuco-PE & 1 & 2 \\
\hline Universidade Federal do Amazonas-AM & 1 & 2 \\
\hline Total & 50 & 100 \\
\hline Internacionais & $n$ & $\%$ \\
\hline Maltepe University, Turquia & 1 & 16,67 \\
\hline Syracuse University, Estados Unidos da América & 1 & 16,67 \\
\hline Uludag University, Turquia & 1 & 16,67 \\
\hline Université de Bourgogne, França & 1 & 16,67 \\
\hline Universidad de La Frontera, Chile & 1 & 16,67 \\
\hline Universidade do Minho, Portugal & 1 & 16,67 \\
\hline Total & 6 & 100 \\
\hline
\end{tabular}

* Excluindo-se resenhas.

A Figura 1 representa a evolução da quantidade de manuscritos submetidos e seu destino no triênio 2007, 2008 e 2009. Nota-se que no ano de 2008 houve um expressivo aumento de processos em decorrência da realização de chamada para recebimento de trabalhos, além da consolidação paulatina da Paideia na base SciELO, o que elevou sua visibilidade no meio acadêmico.

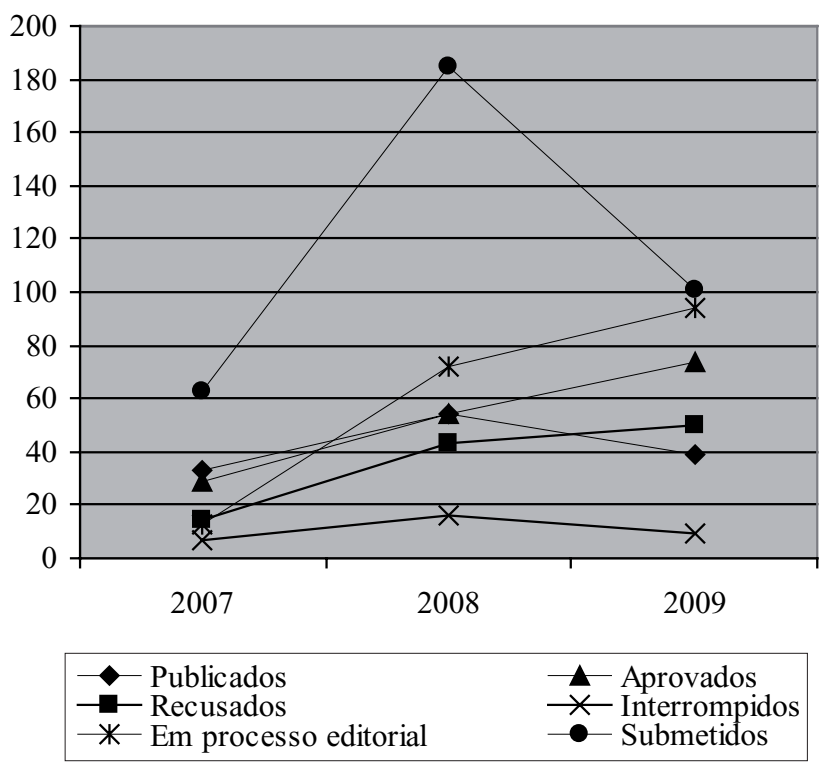

Figura 1. Número de manuscritos submetidos à revista Paideia e seu destino editorial no triênio 2007, 2008 e 2009.

No que concerne à procedência dos consultores $a d h o c$, nota-se o predomínio de revisores lotados em universidades do Estado de São Paulo (67,1\%), que, somados aos avaliadores oriundos de outras instituições pertencentes à região Sudeste, totalizaram $79,4 \%$ do corpo de revisores. A região Sul comportou $11,7 \%$ dos assessores, as regiões Nordeste e Centro-Oeste perfizeram $4,4 \%$ cada uma e a região Norte $1 \%$ (Tabela 4)

Tabela 4

Abrangência do corpo de consultores ad hoc da Paideia conforme origem geográfica

\begin{tabular}{lcc}
\hline \multicolumn{1}{c}{ Estado } & \multicolumn{2}{c}{ Consultores } \\
& n & \%* \\
\hline Região Sudeste & 137 & 67,1 \\
$\quad$ São Paulo & 17 & 8,4 \\
$\quad$ Rio de Janeiro & 5 & 2,4 \\
$\quad$ Minas Gerais & 1 & 0,5 \\
$\quad$ Espírito Santo & 160 & 78,4 \\
Subtotal & & \\
Região Sul & 18 & 8,9 \\
$\quad$ Rio Grande do Sul & 3 & 1,4 \\
Paraná & 3 & 1,4 \\
$\quad$ Santa Catarina & 24 & 11,7 \\
Subtotal & & \\
Região Nordeste & 4 & 1,9 \\
Bahia & 2 & 1,0 \\
Rio Grande do Norte & 1 & 0,5 \\
Pernambuco & & Continua...
\end{tabular}


Tabela 4

Continuação...

\begin{tabular}{lcc}
\hline \multirow{2}{*}{ Estado } & \multicolumn{2}{c}{ Consultores } \\
& n & \%* \\
\hline Paraíba & 1 & 0,5 \\
Ceará & 1 & 0,5 \\
Subtotal & 9 & 4,4 \\
Região Centro-Oeste & & \\
$\quad$ Brasília & 7 & 3,4 \\
$\quad$ Mato Grosso & 2 & 1,0 \\
Subtotal & 9 & 4,4 \\
Região Norte & & \\
$\quad$ Pará & 1 & 0,5 \\
$\quad$ Amazonas & 1 & 0,5 \\
Subtotal & 2 & 1,0 \\
Total & 204 & 100 \\
\hline
\end{tabular}

* Cálculo baseado no total de 204 assessores em 2009.

\section{Considerações finais}

Diante do aumento expressivo da produção científica nacional, os periódicos científicos desempenham um papel fundamental no processo de seleção e qualificação dos artigos, por constituírem os principais veículos de publicação e também os de maior credibilidade.

O presente relatório permitiu mapear o quadro da tramitação editorial da revista Paideia em 2009, evidenciando que, nos últimos anos, a revista vem tomando contornos diferenciados e se fortalecendo de forma gradual e consistente, consolidando-se, paulatinamente, como espaço editorial aglutinador dos conhecimentos produzidos na área da Psicologia $\mathrm{e}$ Educação.

Fica, assim, consignada a expectativa de que as demais publicações da área da Psicologia, Educação e afins possam, igualmente, cultivar uma cultura de divulgação de dados de seus processos de publicação, que permitam delinear suas peculiaridades e, assim, estabelecer eventuais similaridades e distinções.

Quanto às perspectivas de futuro da revista, a Paideia está implantando o processo de submissão eletrônica de manuscritos, a partir da ferramenta Open Journal Systems (OJS), componente da metodologia SciELO, a fim de tornar o sistema de tramitação editorial e publicação mais eficaz. Objetiva-se, ainda, dar prosseguimento ao trabalho de submissão da revista à análise para inclusão em novas bases de dados internacionais.

A somatória desses esforços tem contribuído para um maior reconhecimento da revista Paideia na área da Psicologia, Educação e afins, além de aumentar sua visibilidade em âmbito nacional e internacional. Para consecução das metas traçadas e consolidação das já alcançadas, é necessário que haja alocação de recursos financeiros e de pessoal especializado, além daqueles já dispostos atualmente, a fim de que a revista mantenha seu vigor criativo.

Esperamos continuar mantendo o mesmo empenho e fervor que nos impulsionaram até o momento a prosseguir em direção a novas conquistas. Entre os novos desafios colocados no horizonte figuram a necessidade de ampliar a indexação no exterior, implantar a submissão online e aprimorar os indicadores bibliométricos.

\section{Referências}

Bastos, A. V. B. e cols. (2009). Critérios do qualis de periódicos: Área psicologia. São Paulo: Fundação Coordenação de Aperfeiçoamento de Pessoal de Nível Superior. Recuperado em 15 maio 2009, de http://www. anpepp.org.br/index-aval.htm

Manoel Antônio dos Santos é Professor Doutor da Faculdade de Filosofia, Ciências e Letras de Ribeirão Preto da Universidade de São Paulo, Editor da Paideia. 